\title{
Opposing effects of thyroid hormones on cancer risk: a population-based study
}

\author{
Eilon Krashin 1,2, Barbara Silverman 3,4, David M Steinberg5, Daniel Yekutieli5, Shmuel Giveon ${ }^{6}$, Offer Fabian', \\ Aleck Hercbergs7, Paul J Davis ${ }^{8}$, Martin Ellis 1,9,10 and Osnat Ashur-Fabian (1),2
}

${ }^{1}$ Translational Oncology Laboratory, Meir Medical Center, Kfar-Saba, Israel, ${ }^{2}$ Department of Human Molecular Genetics and Biochemistry, Sackler School of Medicine, Tel Aviv University, Tel Aviv, Israel, ${ }^{3}$ Israel National Cancer Registry, Ministry of Health, Gertner Institute, Sheba Medical Center, Tel Hashomer, Israel, ${ }^{4}$ School of Public Health, Sackler School of Medicine, ${ }^{5}$ Department of Statistics and Operations Research, Tel Aviv University, Tel Aviv, Israel, ${ }^{6} \mathrm{Clalit}$ Health Services, Tel Aviv, Israel, ${ }^{7}$ Department of Radiation Oncology, Cleveland Clinic, Cleveland, Ohio, USA, ${ }^{8}$ Department of Medicine, Albany Medical College, Albany, New York, USA, ${ }^{9}$ Hematology Institute and Blood Bank, Meir Medical Center, Kfar-Saba, Israel, and ${ }^{10}$ Sackler School of Medicine, Tel Aviv University, Tel Aviv, Israel

Correspondence should be addressed to O Ashur-Fabian Email osnataf@gmail.com

\begin{abstract}
Objective: The association between dysregulated thyroid hormone function and cancer risk is inconclusive, especially among different age groups and uncommon malignancies. We sought to determine the relation of TSH and free T4 levels with overall cancer risk as well as risk of specific cancer types.

Design and methods: Data on thyroid hormone profile was collected from 375635 Israeli patients with no prior history of cancer. Cancer cases were identified via the Israel National Cancer Registry. Cox proportional hazards model was used to assess hazard ratios for overall cancer as well as 20 cancer subgroups.

Results: In this study, 23808 cases of cancer were detected over median follow up of 10.9 years. Among patients younger than 50 at inclusion, TSH in the hyperthyroid range, elevated free T4 and subclinical hyperthyroidism were associated with increased cancer risk (HR: 1.3, 1.28 and 1.31, respectively). In contrast, patients 50 or older with clinical hyperthyroidism were at lower cancer risk (HR: 0.64). Elevated TSH was associated with decreased risk of prostate cancer (HR: 0.67). Log-TSH elevation was associated with decreased risk of thyroid cancer (HR: 0.82) and increased risk of melanoma (HR: 1.11) and uterine cancer (HR: 1.27). Elevated free T4 was associated with increased lung cancer risk (HR: 1.54), while free T4 levels above the normal range and clinical hyperthyroidism were related to lower colorectal cancer risk (HR: 0.59 and 0.08, respectively).
\end{abstract}

Conclusions: Thyroid hormones display opposing effects on cancer risk, based on patient age and cancer type.

\section{Introduction}

Thyroid hormones are essential for normal growth and development. Disorders of thyroid function are common, affecting an estimated $5 \%$ of the general population (1) and are known to be positively correlated with patient age (2). Cancer is a leading cause of death worldwide and active research is conducted for the identification of novel cancer risk factors.

Preclinical research has demonstrated an effect of thyroid hormones on cancer growth. Thyroid hormones were shown to induce in vitro proliferation in multiple cancer cell types $(3,4,5,6,7)$ as well as promote in vivo tumor growth $(8,9,10)$ and angiogenesis $(8,10,11$, 12). Data from population-based research support an association between dysregulation of thyroid hormone function and cancer risk $(13,14,15)$. However, the nature of this relationship remains inconclusive and ill-defined. While hyperthyroidism may be related to increased risk of several common malignancies, such as lung, breast (c) 2021 European Society of Endocrinology Printed in Great Britain
Published by Bioscientifica Ltd. 
and prostate cancer $(13,14,15,16,17)$, it may also offer a protective effect for others $(18,19)$. Most population studies relied on measurements of thyroid-stimulating hormone (TSH), the chief regulator of thyroid hormone synthesis, and did not determine the effect of thyroxine (T4), the main hormone produced by the thyroid. Research mainly focused on the most common malignancies and studies were underpowered to assess the association for uncommon cancer types. Moreover, most population studies focused on middle-aged to elderly patients and did not perform a risk analysis for younger patients. As the relative incidence of specific malignancies may vary with age, an analysis of cancer risk in young as well as older patient groups is mandated. Finally, no previous study conducted an adjustment of the statistical analysis for multiple comparisons.

We performed a large exploratory population-based study to determine the relation between TSH and Free T4 (FT4) levels and risk of cancer in different age groups and for multiple cancer types, including assessment of the risk of uncommon malignancies.

\section{Methods}

\section{Study population}

A population-based historical cohort study was performed, including individuals medically insured by Clalit Health Services (CHS), the largest health maintenance organization in Israel, providing healthcare for 4.6 million residents. CHS has a comprehensive integrated electronic medical database, encompassing all medical information regarding primary care and specialist clinic visits, pharmacy purchasing, laboratory tests and hospital admissions. Diagnoses are captured in the registry by means of diagnosis-specific codes, in accordance with the International Classification of Diseases, Ninth Revision (ICD-9). The current study was conducted in the SharonShomron district of Israel, which accounts for $14.7 \%$ of all patients insured by CHS. Adult patients ( $>18$ years) who had a blood test for TSH levels between January 2000 and December 2016 were included. TSH tests are routinely conducted in Israel and one test or more was performed for $68.9 \%$ of the district population insured by CHS during the study period. Patients were included at the date of the first TSH test and followed until a diagnosis of cancer, death or the end of follow up (December 31, 2016). We excluded patients who prior to study inclusion were diagnosed with cancer, had a diagnosis of thyroid function disorder (hypothyroidism or hyperthyroidism, per ICD-9 code) or were treated with thyroid hormone altering medication (levothyroxine, liothyronine, propilthiouracil or methimazole), as well as patients with missing gender data. Based on these criteria, 34734 patients were excluded (Supplementary Fig. 1, see section on supplementary materials given at the end of this article).

\section{Data collection}

All patients had blood tests collected for TSH. For FT4, tests available on the date of inclusion were also collected. Data on covariates were collected at baseline and included age, gender, ethnicity (Jewish or non-Jewish), socioeconomic status (low, medium or high income), BMI, smoking status, comorbidities (hypertension, dyslipidemia, diabetes mellitus, ischemic heart disease, congestive heart failure, cerebrovascular disease, chronic renal failure, chronic lung disease, chronic liver disease) and thyroid function disorder (hyperthyroidism or hypothyroidism). Data on thyroid hormone altering medication use was collected throughout the study period. Socioeconomic status was defined based on patient's neighborhood of residence. Comorbidities were defined based on ICD-9 codes. Thyroid hormone altering treatment was defined as at least one prescription issued of medication affecting the thyroid hormone axis. Relevant ICD-9 codes are detailed in Supplementary Table 1.

\section{Laboratory measurements}

Serum TSH and FT4 levels were measured at the CHS central hormone laboratory using a commercial RIA kit (RIA-gnost ${ }^{\circledR}$ hTSH and free T4, Schering-Cis Bio International, Gif-sur-Yvette, France) with lower limits of detection of $0.01 \mathrm{mIU} / \mathrm{L}$ and $0.06 \mathrm{ng} / \mathrm{dL}$, respectively. The normal range was $0.55-4.78 \mathrm{mIU} / \mathrm{L}$ for TSH and 0.78-1.55 $\mathrm{ng} / \mathrm{dL}$ for FT4.

\section{Cancer diagnosis}

Data on cancer cases were obtained from the Israel National Cancer Registry (INCR), a pathology-based registry covering the entire Israeli population. Reporting to the INCR has been mandatory since 1982. Data are collected on all malignant neoplasms except basal and squamous cell carcinomas of the skin. Cancer site and morphology are coded according to the codes of the International Classification of Diseases for Oncology, Third Edition (ICD-O-3). Linking patients to the cancer registry 
database was conducted using the unique nine-digit national identification number and was authenticated by first and last name, date of birth and gender. Cancer occurrence was defined as any malignancy (invasive or in situ) diagnosed during the study period. Cancer cases were divided into 20 cancer subgroups, defined according to ICD-O-3 topography codes. Cancer site classification is detailed in Supplementary Table 2.

\section{Statistical analysis}

Missing data was imputed using the Markov Chain Monte Carlo method to create five imputed datasets that were pooled for analysis. Missingness was $<10 \%$ for all covariates, except for BMI which was $<15 \%$. Multivariable Cox proportional hazard models were constructed to assess the association between thyroid hormones and the risk of cancer. Hazard ratios (HRs) with their 95\% CI were determined. Log-log survival curves established that the proportional hazards assumption was met. All models were adjusted for age, gender, smoking status, socioeconomic state, ethnicity, BMI and comorbidities. Analyses were performed separately for TSH and FT4. Risk was determined for both continuous and categorical representations of hormone levels. Due to the skewed distribution of TSH levels, log-transformed data was used for the continuous analysis. For categorical analysis, TSH and FT4 were respectively divided into five groups: three categories within the normal range, one below the normal range and one above the normal range. The largest population group served as reference: the lowest tertile within the normal range for TSH and the middle tertile for FT4. Trends were determined using Spearman correlation between five equally sized TSH and free T4 groups and the multivariate-adjusted HRs, with the lowest level serving as reference. In addition, five thyroid function groups were defined based on both TSH and FT4 levels: Euthyroidism (FT4 and TSH within the normal range), clinical hypothyroidism (FT4 below normal range and TSH above the normal range), subclinical hyperthyroidism (FT4 within normal range and $\mathrm{TSH}$ above the normal range), clinical hyperthyroidism (FT4 above normal range and TSH below the normal range) and subclinical hypothyroidism (FT4 within normal range and TSH below the normal range), with the euthyroid groups serving as reference.

Analysis was conducted for overall cancer as well as the 20 predefined cancer subgroups. To allow risk assessment for multiple malignancies while minimizing the risk of false-positivity, a stepwise analysis was performed. The omnibus test of model coefficients (based on Chi-square test) was first conducted to determine the significance for inclusion of the respective thyroid hormone variables (TSH, FT4 or thyroid function groups) in the cancer risk model. Benjamini-Hochberg $(\mathrm{BH})$ correction for the resulting $P$-values was then performed to control the false discovery rate (FDR). BH correction, introduced in 1995, allows for effective control of type I errors in multiple testing scenarios (20). Adjusted hazard ratios based on the Cox model were determined only for cancer groups significant at $P<0.1$ following FDR correction. Significance was set at $P<0.05$ for the final analysis. Two subgroup analyses were conducted, one based on gender and another based on patient age at inclusion. As previous studies tended to include middle-aged and older patients $(13,14)$, we defined two age groups above and below 50 years at inclusion in order to separately assess the risk for young and older adults. Finally, four sensitivity analyses were conducted: excluding the first 2 years of follow up in order to eliminate reverse causality, excluding patients treated with thyroid hormone altering medications during the study period, excluding patients with thyroid cancer and excluding patients with missing data.

All statistical analyses were performed using the SPSS software (version 25, SPSS Inc, Chicago, Ill), except for FDR correction which was performed using $\mathrm{R}$ (version 3.6.0, R Foundation for Statistical Computing, Vienna, Austria).

The study was approved by the CHS ethics committee in accordance with the Declaration of Helsinki.

\section{Results}

The study population consisted of 375635 patients who underwent a thyroid hormone test during the study period. All included patients underwent TSH testing, and 110607 patients (29.4\%) had FT4 levels. At inclusion, the majority of patients (91.7\%) had TSH values within the normal reference range. A higher proportion of patients were women (54.9\%), especially at TSH levels above and below the normal reference range, reflecting increased incidence of thyroid disorders in this gender group. Median age at inclusion was 43 (interquartile range, 30-58). Sixty-one percent (229 830 patients) were younger than 50 at inclusion. The most common comorbidities were dyslipidemia (43\%), hypertension (25.5\%), diabetes mellitus (16.1\%) and ischemic heart disease (11.7\%) (Table 1). Compared with persons with a TSH level in the normal range, patients in the higher and lower TSH categories 
Table 1 Baseline characteristics of study participants. Data are presented as $n(\%)$ or as median (IQR).

\begin{tabular}{lc}
\hline Variables & Values \\
\cline { 2 - 2 } TSH, mIU/L & 375635 \\
Age, years & $1.90(1.32-2.72)$ \\
Follow up years & $43(30-58)$ \\
BMI, kg/m² & $10.9(6.7-13.6)$ \\
Women & $26.7(23.5-30.7)$ \\
Dyslipidemia & $206081(54.9 \%)$ \\
Hypertension & $160563(42.7 \%)$ \\
Diabetes mellitus & $95865(25.5 \%)$ \\
Ischemic heart disease & $60535(16.1 \%)$ \\
Congestive heart failure & $43907(11.7 \%)$ \\
Chronic lung disease & $13073(3.5 \%)$ \\
Cerebrovascular disease & $11689(3.1 \%)$ \\
Chronic renal failure & $10458(2.8 \%)$ \\
Chronic liver disease & $10358(2.8 \%)$ \\
Smoking & $1019(0.3 \%)$ \\
Ethnicity & $105592(28.1 \%)$ \\
$\quad$ Jewish & \\
$\quad$ Non-Jewish & $287713(76.7 \%)$ \\
Socioeconomic status & $87172(23.3 \%)$ \\
$\quad$ Low income & \\
$\quad$ Middle income & \\
$\quad$ High income & $110392(32.3 \%)$ \\
Thyroid medication & $130922(38.4 \%)$ \\
$\quad$ Antithyroid treatment & $100143(29.3 \%)$ \\
$\quad$ Thyroid replacement & $1780(0.5 \%)$ \\
\hline & $21231(5.6 \%)$ \\
\hline &
\end{tabular}

$\mathrm{IQR}$, interquartile range; TSH, thyroid stimulating hormone.

had higher rates of comorbidities (Supplementary Table 3). For 17776 patients with available data of 3-12 months following the initial test, who did not receive thyroid altering medication, a significant consistency existed for thyroid function group (Spearmen's r, 0.67; $P<0.0001$ ). Over a median follow up of 10.9 years, 23808 cases of cancer were detected throughout 3687710 person-years of follow up, affecting $6.3 \%$ of the study population. Sitespecific cancer cases by age groups and TSH strata are detailed in Supplementary Table 4.

\section{The association of TSH levels with cancer risk is dependent on age and tumor type}

The first aim of this study was to identify associations between TSH levels and cancer risk. There was no significant association between log-TSH levels and overall risk of cancer (Table 2). A significant interaction exists between TSH and age in the cancer risk model $(P<0.001)$. Patients younger than 50 at inclusion (Table 3 and Supplementary Table 5) with TSH in the hyperthyroid range $(<0.55 \mathrm{mIU} / \mathrm{L})$ were at increased overall risk of cancer, compared with the reference group (adjusted HR: 1.3; 95\% CI: 1.12-1.51). Following FDR correction, inclusion of TSH levels in the cancer risk model was significant for prostate cancer, thyroid cancer, melanoma and uterine cancer (Supplementary Table 6) and hazard ratios were determined for these cancer subtypes (Table 2). Patients with TSH in the hypothyroid range ( $>4.78$ $\mathrm{mIU} / \mathrm{L}$ ) were at significantly lower risk of prostate cancer (adjusted HR: 0.67; 95\% CI: 0.54 to 0.85). The risk of thyroid cancer decreased with increasing $\log$-TSH levels (adjusted HR: 0.82 ; 95\% CI: 0.75 to 0.9 per unit logTSH increase) and doubled in patients with TSH in the hyperthyroid range (adjusted HR: 2.01, 95\% CI: 1.74 to 2.32). However, a significant trend for thyroid cancer risk was not demonstrated $(P=0.5)$. Log-TSH increase was associated with increased risk of melanoma (adjusted HR:, 1.11 ; $95 \%$ CI: 1.02 to $1.20, P$ for trend $=0.04$ ) and uterine cancer (adjusted HR: 1.27; 95\% CI: 1.13 to 1.41, $P$ for trend $<0.0001)$. For both malignancies, this effect was mainly driven by increased risk within the normal TSH range. Sensitivity analyses corroborated our findings (Supplementary Table 7). Specifically, results remained significant following exclusion of first 2 years of follow up, suggesting the demonstrated relations were not related to reverse causality. Notably, prostate cancer was the only malignancy for which the association was lost following the exclusion of thyroid altering medication use during the study period.

\section{Free T4 differentially affects cancer risk based on age and malignancy type}

We next sought to examine the association between FT4 and cancer risk. Following FDR correction, inclusion of FT4 levels in the cancer risk model was significant for overall cancer as well as for lung cancer and colorectal cancer (Supplementary Table 6). Hazard ratios were determined for overall cancer and both cancer subtypes (Table 4). Overall risk of cancer was slightly higher for patients with FT4 in the upper tertile of the normal range (adjusted HR: 1.1; 95\% CI: 1.03-1.17). A significant interaction was demonstrated between FT4 and age in the cancer risk model $(P=0.003)$. In patients younger than 50, elevated FT4 was associated with an increased overall cancer risk (adjusted HR: 1.28; 95\% CI: 1.1-1.49 per unit FT4 increase), while patients 50 or older (Table 3) with hyperthyroxinemia (FT4 $>1.55 \mathrm{ng} / \mathrm{dL}$ ) were at lower cancer risk (adjusted HR: 0.87; 95\% CI: 0.76-0.99). Elevated FT4 was associated with increased lung cancer risk (adjusted HR:, 1.54 ; 95\% CI: $1.1-2.03, P$ for trend $=0.04$ ), an effect driven mainly by increased risk within the normal FT4 range. Patients with hyperthyroxinemia had significantly 
Table 2 Hazard ratios for overall and site-specific cancers by TSH strata. HRs are adjusted for age, gender, smoking status, socioeconomic state, ethnicity, BMI and comorbidities (hypertension, dyslipidemia, diabetes mellitus, ischemic heart disease, congestive heart failure, cerebrovascular disease, chronic renal failure, chronic lung disease, chronic liver disease).

\begin{tabular}{l}
\hline Malignancy/TSH strata (mIU/L) \\
\hline Any cancer ${ }^{\mathrm{a}}$ \\
$<0.55$ \\
$0.55-1.96$ \\
$1.96-3.37$ \\
$3.37-4.78$ \\
$>4.78$ \\
Prostate cancer ${ }^{\mathrm{fb}}$ \\
$<0.55$ \\
$0.55-1.96$ \\
$1.96-3.37$ \\
$3.37-4.78$ \\
$>4.78$ \\
Thyroid cancer ${ }^{\mathrm{jc}}$ \\
$<0.55$ \\
$0.55-1.96$ \\
$1.96-3.37$ \\
$3.37-4.78$ \\
$>4.78$ \\
Melanoma ${ }^{\text {fd }}$ \\
$<0.55$ \\
$0.55-1.96$ \\
$1.96-3.37$ \\
$3.37-4.78$ \\
$>4.78$ \\
Uterine cancer ${ }^{\mathrm{je}}$ \\
$<0.55$ \\
$0.55-1.96$ \\
$1.96-3.37$ \\
$3.37-4.78$ \\
$>4.78$ \\
\hline
\end{tabular}

\begin{tabular}{r}
\hline Events $(n)$ \\
\hline 23808 \\
893 \\
11623 \\
7657 \\
2243 \\
1392 \\
2406 \\
77 \\
1320 \\
767 \\
165 \\
77 \\
866 \\
55 \\
422 \\
243 \\
96 \\
50 \\
1189 \\
31 \\
523 \\
430 \\
138 \\
67 \\
617 \\
14 \\
238 \\
232 \\
84 \\
49 \\
\end{tabular}

$\begin{array}{r}\hline \text { Participants }(n) \\ \hline 375635 \\ 11338 \\ 186116 \\ 123474 \\ 34860 \\ 19847 \\ 169554 \\ 4189 \\ 90860 \\ 54838 \\ 13267 \\ 6400 \\ 375635 \\ 11338 \\ 186116 \\ 123474 \\ 34860 \\ 19847 \\ 375635 \\ 11338 \\ 186116 \\ 123474 \\ 34860 \\ 19847 \\ 206081 \\ 7149 \\ 95256 \\ 68636 \\ 21593 \\ 13447 \\ \\ \hline 64\end{array}$

\begin{tabular}{l}
\multicolumn{1}{c}{ HR $(95 \% \mathrm{CI})$} \\
\hline $1.00(0.98-1.02)^{\dagger}$ \\
$0.99(0.98-1.06)$ \\
Reference \\
$1.04(1.01-1.07)$ \\
$1.02(0.98-1.07)$ \\
$0.97(0.91-1.02)$ \\
$0.96(0.91-1.02)^{\dagger}$ \\
$0.90(0.71-1.13)$ \\
Reference \\
$1.03(0.94-1.12)$ \\
$0.87(0.75-1.01)$ \\
$0.67(0.54-0.85)$ \\
$0.82(0.75-0.90)^{\dagger}$ \\
$2.01(1.74-2.32)$ \\
Reference \\
$0.83(0.71-0.97)$ \\
$1.10(0.88-1.37)$ \\
$0.95(0.70-1.29)$ \\
$1.11(1.02-1.20)^{\dagger}$ \\
$0.89(0.74-1.08)$ \\
Reference \\
$1.25(1.10-1.43)$ \\
$1.39(1.16-1.67)$ \\
$1.09(0.84-1.40)$ \\
$1.27(1.13-1.41)^{\dagger}$ \\
$0.66(0.39-1.14)$ \\
Reference \\
$1.36(1.13-1.63)$ \\
$1.49(1.16-1.91)$ \\
$1.25(0.92-1.70)$ \\
\\
\hline
\end{tabular}

\begin{tabular}{l}
\hline P-value \\
\hline 0.95 \\
0.79 \\
Reference \\
0.02 \\
0.28 \\
0.23 \\
0.25 \\
0.35 \\
Reference \\
0.56 \\
0.09 \\
0.001 \\
$<0.0001$ \\
$<0.0001$ \\
Reference \\
0.02 \\
0.40 \\
0.75 \\
0.02 \\
0.55 \\
Reference \\
0.001 \\
0.001 \\
0.53 \\
$<0.0001$ \\
0.13 \\
Reference \\
0.001 \\
0.001 \\
0.15 \\
\end{tabular}

SSignificant in FDR-adjusted model (Supplementary Table 5); ${ }^{\dagger}$ HR per unit log-TSH increase; $P$ for trend: ${ }^{a} 0.75 ;{ }^{b} 0.87 ;{ }^{c} 0.5 ;{ }^{d} 0.04 ;{ }^{e}<0.0001$. $\mathrm{HR}$, hazard ratio; $\mathrm{TSH}$, thyroid stimulating hormone.

lower risk of colorectal cancer (adjusted HR: 0.59; 95\% CI: 0.41-0.85). Subgroups analysis suggested this effect was most prominent in women and patients 50 and older (Supplementary Table 8). The associations for lung cancer and colorectal cancer remained significant following exclusion of first 2 years of follow up (Supplementary Table 9).

\section{Hyperthyroidism is associated with reduced overall and colorectal cancer risk}

Lastly, we assessed the correlation between thyroid function status and cancer risk. Following FDR correction, inclusion of thyroid function groups in the cancer risk model was significant for colorectal cancer and hematological malignancies (Supplementary Table 6). Hazard ratios were determined for overall cancer risk and both cancer subtypes (Table 5). Analysis indicated a reduction in the overall risk of cancer among patients with clinical hyperthyroidism (adjusted HR: 0.68; 95\% CI: 0.52-0.89). There was a significant interaction between thyroid function and age in the overall cancer risk model $(P=0.001)$, yet not for specific cancer types. Patients younger than 50 with subclinical hyperthyroidism (Table 3 and Supplementary Table 10) had higher overall risk of cancer (adjusted HR: 1.31; 95\% CI: 1.06-1.61), while patients aged 50 or older with clinical hyperthyroidism had lower cancer risk (adjusted HR: 0.64; 95\% CI: 0.470.87). Clinical hyperthyroidism was associated with lower risk of colorectal cancer (adjusted HR: 0.08; 95\% CI: 0.010.60). No association was demonstrated between thyroid function and risk of hematological malignancies in the final analysis. Results were similar following exclusion of first 2 years of follow-up, thyroid altering medication use during the study period (Supplementary Table 11) and thyroid cancer patients (Supplementary Table 12). 


\section{Discussion}

In this exploratory population-based study, we have demonstrated associations between thyroid hormone levels and cancer risk that are tumor-specific and agedependent. These associations were driven by alterations in thyroid hormone levels not only above and below the reference range but also within the normal range.

The relation between thyroid hormones and cancer risk has been examined in several population studies $(13,14,15)$. However, these studies included relatively small patient cohorts, limiting the analysis to common cancer types, and did not present stratification by age. Our large study was sufficiently powered for assessment of risk for multiple cancer subtypes. As cancer is an agerelated disease, we also performed separate analyses for different age groups. The use of FDR correction for multiple comparisons decreased the likelihood of falsepositivity and validated the demonstrated relations. Furthermore, adjustment for multiple clinical parameters and utilization of several sensitivity analyses decreased the likelihood of bias. Finally, analysis of the full spectrum of TSH and FT4, for both continuous and categorical ranges, allowed determination of hormone effects above, below and within the normal range. Collectively, the design of our study enabled us to identify novel associations for both common and uncommon malignancies and to determine differing effects of thyroid hormones on cancer risk based on age. Specifically, our analysis suggests that hyperthyroid function is associated with increased cancer risk in younger patients but may have a protective effect in older patients. As the tumor promoting effect of thyroid hormones appears to be site-specific, these results may signify the differing incidence of dominant cancer types in different age groups. In particular, a relatively high incidence of thyroid cancer in the younger patient group and a high incidence of colorectal cancer in the older age group (21). The demonstrated interaction between age and thyroid hormones for overall cancer risk, yet not for the risk of any specific malignancy, supports this hypothesis.

In our study, hyperthyroid TSH levels were associated with increased risk of thyroid cancer and elevated FT4 with increased lung cancer risk. Correspondingly, hypothyroid TSH levels were related to decreased risk of prostate cancer. Associations remained significant for 2 years and more prior to cancer diagnosis, further supporting a causal relation. For lung cancer, a positive trend was demonstrated, suggesting a dose-dependent effect of FT4. As the degree of TSH change relative to free T4 varies 
Table 4 Hazard ratios for overall and site-specific cancers by free T4 (FT4) strata. HRs are adjusted for age, gender, smoking status, socioeconomic state, ethnicity, BMI, and comorbidities (hypertension, dyslipidemia, diabetes mellitus, ischemic heart disease, congestive heart failure, cerebrovascular disease, chronic renal failure, chronic lung disease, chronic liver disease).

\begin{tabular}{l}
\hline Malignancy/FT4 strata $(\mathrm{ng} / \mathrm{dL})$ \\
\hline Any cancer ${ }^{\mathrm{ja}}$ \\
$<0.78$ \\
$0.78-1.04$ \\
$1.04-1.3$ \\
$1.3-1.55$ \\
$>1.55$ \\
Lung cancer $r^{\mathrm{jb}}$ \\
$<0.78$ \\
$0.78-1.04$ \\
$1.04-1.3$ \\
$1.3-1.55$ \\
$>1.55$ \\
Colorectal cancer ${ }^{\mathrm{fc}}$ \\
$<0.78$ \\
$0.78-1.04$ \\
$1.04-1.3$ \\
$1.3-1.55$ \\
$>1.55$
\end{tabular}

\begin{tabular}{c}
\hline Events $(n)$ \\
\hline 6718 \\
159 \\
1940 \\
2983 \\
1349 \\
287 \\
583 \\
20 \\
136 \\
252 \\
142 \\
33 \\
890 \\
18 \\
247 \\
433 \\
161 \\
31
\end{tabular}

\begin{tabular}{c}
\hline Participants $(n)$ \\
\hline 110607 \\
2482 \\
34203 \\
52932 \\
17205 \\
3785 \\
110607 \\
2482 \\
34203 \\
52932 \\
17205 \\
3785 \\
110607 \\
2482 \\
34203 \\
52932 \\
17205 \\
3785
\end{tabular}

\begin{tabular}{l}
\multicolumn{1}{c}{$\mathbf{H R}(95 \% \mathrm{Cl})$} \\
\hline $1.05(0.95-1.15)^{\ddagger}$ \\
$0.90(0.76-1.05)$ \\
$1.00(0.94-1.06)$ \\
Reference \\
$1.10(1.03-1.17)$ \\
$0.89(0.78-1.00)$ \\
$1.54(1.16-2.03)^{\ddagger}$ \\
$1.38(0.87-2.18)$ \\
$0.87(0.71-1.07)$ \\
Reference \\
$1.29(1.04-1.58)$ \\
$1.20(0.83-1.73)$ \\
$0.82(0.62-1.09)^{\ddagger}$ \\
$0.67(0.42-1.07)$ \\
$0.87(0.76-1.01)$ \\
Reference \\
$0.85(0.71-1.02)$ \\
$0.59(0.41-0.85)$ \\
\hline
\end{tabular}

\begin{tabular}{l}
\multicolumn{1}{c}{ P-value } \\
\hline 0.38 \\
0.19 \\
1.00 \\
Reference \\
0.005 \\
0.05 \\
0.002 \\
0.17 \\
0.19 \\
Reference \\
0.02 \\
0.33 \\
0.17 \\
1.00 \\
0.09 \\
Reference \\
0.07 \\
0.004 \\
\hline
\end{tabular}

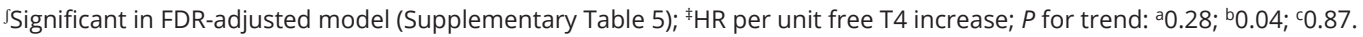

$H R$, hazard ratio; T4, thyroxine.

significantly among individuals (22), a corresponding association for TSH may not be demonstrated. Our results are in accordance with previous research on lung $(13,14)$, thyroid $(23,24)$ and prostate cancer $(13,15,25)$. To the best of our knowledge, this is the first population study to suggest a relation between hyperthyroidism, based on
TSH blood levels and thyroid cancer risk. However, as hyperthyroid patients are more likely to undergo thyroid imaging, diagnostic work-up bias cannot be ruled out. The growth promoting effects of thyroid hormones in cancer were demonstrated in large body of preclinical research. T4 was shown to stimulate lung $(6,7,8,26)$,

Table 5 Hazard ratios for overall and site-specific cancers by thyroid function groups. HRs are adjusted for age, gender, smoking status, socioeconomic state, ethnicity, BMI, and comorbidities (hypertension, dyslipidemia, diabetes mellitus, ischemic heart disease, congestive heart failure, cerebrovascular disease, chronic renal failure, chronic lung disease, chronic liver disease).

\begin{tabular}{|c|c|c|c|c|}
\hline Malignancy/thyroid function group & Events $(n)$ & Participants $(n)$ & HR $(95 \% \mathrm{Cl})$ & P-value \\
\hline \multicolumn{5}{|l|}{ Any cancers } \\
\hline Hyperthyroidism & 53 & 840 & $0.68(0.52-0.89)$ & 0.005 \\
\hline SC hyperthyroidism & 475 & 5972 & $1.09(0.99-1.20)$ & 0.06 \\
\hline Euthyroidism & 4880 & 84490 & Reference & Reference \\
\hline SC hypothyroidism & 917 & 13878 & $1.01(0.94-1.09)$ & 0.72 \\
\hline Hypothyroidism & 99 & 1355 & $0.93(0.76-1.13)$ & 0.45 \\
\hline \multicolumn{5}{|l|}{ Colorectal cancer ${ }^{\varsigma}$} \\
\hline Hyperthyroidism & 1 & 840 & $0.08(0.01-0.60)$ & 0.01 \\
\hline SC hyperthyroidism & 57 & 5972 & $0.93(0.71-1.22)$ & 0.58 \\
\hline Euthyroidism & 648 & 84490 & Reference & Reference \\
\hline SC hypothyroidism & 136 & 13878 & $1.10(0.92-1.33)$ & 0.30 \\
\hline Hypothyroidism & 13 & 1355 & $0.88(0.51-1.52)$ & 0.64 \\
\hline \multicolumn{5}{|l|}{ Hematological malignancy } \\
\hline Hyperthyroidism & 1 & 840 & $0.14(0.02-1.02)$ & 0.05 \\
\hline SC hyperthyroidism & 46 & 5972 & $1.20(0.88-1.63)$ & 0.25 \\
\hline Euthyroidism & 430 & 84490 & Reference & Reference \\
\hline SC hypothyroidism & 93 & 13878 & $1.20(0.96-1.51)$ & 0.11 \\
\hline Hypothyroidism & 7 & 1355 & $0.76(0.36-1.61)$ & 0.48 \\
\hline
\end{tabular}


thyroid (27) and prostate (28) cancer growth, while a hypothyroid environment slowed tumor growth $(29,30$, 31 ). One of the main mechanisms mediating these effects is thyroid hormone binding to the plasma membrane integrin $\alpha v \beta 3$, leading to cancer cell proliferation and tumor angiogenesis (32).

Our study has also demonstrated an inverse relation between thyroid function and the risk of specific malignancies. Hyperthyroid patients were at significantly lower risk of colorectal cancer. This finding is in accordance with previous works on thyroid function and colorectal cancer risk $(18,33,34)$, as well as with studies demonstrating a protective effect of levothyroxine treatment on colorectal cancer risk (35, $36,37)$. However, our study is the first to demonstrate the effect of hyperthyroid function on colorectal cancer risk irrespective of thyroid altering medication use. We further identified that TSH in the hypothyroid range was associated with increased risk of uterine cancer and melanoma. Notably, as these associations remained significant for 2 years and more prior to cancer diagnosis, they cannot be attributed to thyroid hormone dysfunction secondary to the malignancy, such as the sick euthyroid syndrome. These results are novel, with only a single study suggesting that hypothyroidism is related to poor uterine cancer outcomes (38). The biological basis for these effects is not well-defined. Treatment of colorectal cancer cells with thyroid hormones induces differentiation and leads to reduced proliferation (39, 40). Both endometrial tissue (41) and melanocytes (42) express the TSH receptor and a direct growth-promoting effect of TSH may be involved in the pathogenesis of uterine cancer and melanoma.

For several malignancies, including lung cancer, uterine cancer and melanoma, risk appeared to be driven by changes within the reference range. These results are in-line with a growing body of research suggesting changes of thyroid hormones within the normal range are associated with adverse clinical outcomes, including fractures $(43,44)$, cardiovascular outcomes $(45,46)$ and adverse lipid profile (47). Our findings suggest that for at-risk patients, there may be benefit to maintain TSH and free $\mathrm{T} 4$ levels with a narrow range.

This study has several limitations that need to be considered. Our analysis was based on thyroid hormone measurements at a specific time point, and we could not determine the effect of changes in thyroid hormone levels over time. Moreover, specifically for breast cancer, we were unable to adjust for important risk factors, including menopausal status and female hormone use. This may explain why our analysis did not demonstrate an association between thyroid hormones and breast cancer risk, which was noted in previous populationbased studies $(14,16,17,48)$. We also could not adjust for radioiodine therapy use, which could affect thyroid cancer risk (49) and were unable to differentiate between primary and secondary thyroid disorders. Finally, our historical cohort study consisted of patients who had underwent thyroid hormone tests and may be potentially biased toward cases with thyroid dysfunction. However, as TSH tests are routinely conducted in Israel and the majority of our patients had thyroid hormone values within the normal range, our finding may be representative of the general population.

To conclude, this study demonstrates opposing effects of thyroid hormones on cancer risk, based on patient age and specific cancer types. These findings mandate further studies and suggest a rationale for future inclusion of thyroid hormone profile in cancer risk assessment of individual patients.

\section{Supplementary materials}

This is linked to the online version of the paper at https://doi.org/10.1530/ EJE-20-1123.

\section{Declaration of interest}

The authors declare that there is no conflict of interest that could be perceived as prejudicing the impartiality of this study.

\section{Funding}

This study received a research grant from the Tel Aviv University Data Science Center, Israel (grant \#0603619891).

\section{Author contribution statement}

E K designed the study and collected, analyzed, and interpreted the data. B S provided the information on cancer diagnoses. D M S and D Y assisted with statistical analysis. S G assisted in data collection. O F assisted in database establishment. A H, P J D and M E assisted in interpretation of the clinical data. O A F designed the study and analyzed and interpreted the data. E K and O A F wrote the manuscript. All authors read and approved the manuscript.

\section{Acknowledgements}

The work of $\mathrm{E} \mathrm{K}$ was done in partial fulfillment of the requirements for a PhD degree from the Sackler Faculty of Medicine, Tel Aviv University, Israel. The authors thank Dr Mira Manor from CHS Central Lab for providing information on laboratory methods. 


\section{References}

1 Antonelli A, Ferrari SM, Corrado A, Di Domenicantonio A \& Fallahi P. Autoimmune thyroid disorders. Autoimmunity Reviews 2015 14 174-180. (https://doi.org/10.1016/j.autrev.2014.10.016)

2 Vanderpump MP. The epidemiology of thyroid disease. British Medical Bulletin 201199 39-51. (https://doi.org/10.1093/bmb/ldr030)

3 Hall LC, Salazar EP, Kane SR \& Liu N. Effects of thyroid hormones on human breast cancer cell proliferation. Journal of Steroid Biochemistry and Molecular Biology 2008109 57-66. (https://doi.org/10.1016/j. jsbmb.2007.12.008)

4 Dinda S, Sanchez A \& Moudgil V. Estrogen-like effects of thyroid hormone on the regulation of tumor suppressor proteins, p53 and retinoblastoma, in breast cancer cells. Oncogene 200221 761-768. (https://doi.org/10.1038/sj.onc.1205136)

5 Tang HY, Lin HY, Zhang S, Davis FB \& Davis PJ. Thyroid hormone causes mitogen-activated protein kinase-dependent phosphorylation of the nuclear estrogen receptor. Endocrinology 2004145 3265-3272. (https://doi.org/10.1210/en.2004-0308)

6 Meng R, Tang HY, Westfall J, London D, Cao JH, Mousa SA, Luidens M, Hercbergs A, Davis FB, Davis PJ et al. Crosstalk between integrin $\alpha v \beta 3$ and estrogen receptor- $\alpha$ is involved in thyroid hormone-induced proliferation in human lung carcinoma cells. PLoS ONE 20116 e27547. (https://doi.org/10.1371/journal.pone.0027547)

7 Mousa SA, Yalcin M, Bharali DJ, Meng R, Tang HY, Lin HY, Davis FB \& Davis PJ. Tetraiodothyroacetic acid and its nanoformulation inhibit thyroid hormone stimulation of non-small cell lung cancer cells in vitro and its growth in xenografts. Lung Cancer 201276 39-45. (https://doi.org/10.1016/j.lungcan.2011.10.003)

8 Carmona-Cortes J, Rodriguez-Gomez I, Wangensteen R, Banegas I, Garcia-Lora ÁM, Quesada A, Osuna A \& Vargas F. Effect of thyroid hormone-nitric oxide interaction on tumor growth, angiogenesis, and aminopeptidase activity in mice. Tumour Biology 201435 5519-5526. (https://doi.org/10.1007/s13277-014-1726-2)

9 Vermey ML, Marks GT \& Baldridge MG. Effect of thyroid function on MNU-induced mammary carcinogenesis. Zoological Science 201532 272-277. (https://doi.org/10.2108/zs140124)

10 Sterle HA, Valli E, Cayrol F, Paulazo MA, Martinel Lamas DJ, Diaz Flaque MC, Klecha AJ, Colombo L, Medina VA, Cremaschi GA et al. Thyroid status modulates $\mathrm{T}$ lymphoma growth via cell cycle regulatory proteins and angiogenesis. Journal of Endocrinology 2014 222 243-255. (https://doi.org/10.1530/JOE-14-0159)

11 Cayrol F, Diaz Flaque MC, Fernando T, Yang SN, Sterle HA, Bolontrade M, Amoros M, Isse B, Farias RN, Ahn H et al. Integrin $\alpha v \beta 3$ acting as membrane receptor for thyroid hormones mediates angiogenesis in malignant T cells. Blood 2015125 841-851. (https:// doi.org/10.1182/blood-2014-07-587337)

12 Mousa SA, Davis FB, Mohamed S, Davis PJ \& Feng X. Proangiogenesis action of thyroid hormone and analogs in a threedimensional in vitro microvascular endothelial sprouting model. International Angiology 200625 407-413.

13 Hellevik AI, Asvold BO, Bjoro T, Romundstad PR, Nilsen TI \& Vatten LJ. Thyroid function and cancer risk: a prospective population study. Cancer Epidemiology, Biomarkers and Prevention 200918 570-574. (https://doi.org/10.1158/1055-9965.EPI-08-0911)

14 Khan SR, Chaker L, Ruiter R, Aerts JG, Hofman A, Dehghan A, Franco OH, Stricker BH \& Peeters RP. Thyroid function and cancer risk: the Rotterdam study. Journal of Clinical Endocrinology and Metabolism 2016101 5030-5036. (https://doi.org/10.1210/jc.20162104)

15 Chan YX, Knuiman MW, Divitini ML, Brown SJ, Walsh J \& Yeap BB. Lower TSH and higher free thyroxine predict incidence of prostate but not breast, colorectal or lung cancer. European Journal of Endocrinology 2017177 297-308. (https://doi.org/10.1530/EJE-170197)

16 Tosovic A, Becker C, Bondeson AG, Bondeson L, Ericsson UB, Malm J \& Manjer J. Prospectively measured thyroid hormones and thyroid peroxidase antibodies in relation to breast cancer risk. International Journal of Cancer 2012131 2126-2133. (https://doi.org/10.1002/ ijc.27470)

17 Sogaard M, Farkas DK, Ehrenstein V, Jorgensen JO, Dekkers OM \& Sorensen HT. Hypothyroidism and hyperthyroidism and breast cancer risk: a nationwide cohort study. European Journal of Endocrinology 2016174 409-414. (https://doi.org/10.1530/EJE-150989)

18 Chan YX, Alfonso H, Chubb SA, Fegan PG, Hankey GJ, Golledge J, Flicker L \& Yeap BB. Higher thyrotropin concentration is associated with increased incidence of colorectal cancer in older men. Clinical Endocrinology 201786 278-285. (https://doi.org/10.1111/cen.13271)

19 Hassan MM, Kaseb A, Li D, Patt YZ, Vauthey JN, Thomas MB, Curley SA, Spitz MR, Sherman SI, Abdalla EK et al. Association between hypothyroidism and hepatocellular carcinoma: a casecontrol study in the United States. Hepatology 200949 1563-1570. (https://doi.org/10.1002/hep.22793)

20 Benjamini Y \& Hochberg Y. Controlling the false discovery rate: a practical and powerful approach to multiple testing. Journal of the Royal Statistical Society: Series B 199557 289-300. (https://doi. org/10.1111/j.2517-6161.1995.tb02031.x)

21 Bray F, Ferlay J, Soerjomataram I, Siegel RL, Torre LA \& Jemal A. Global cancer statistics 2018: GLOBOCAN estimates of incidence and mortality worldwide for 36 cancers in 185 countries. CA: A Cancer Journal for Clinicians 201868 394-424. (https://doi.org/10.3322/ caac.21492)

22 Hadlow NC, Rothacker KM, Wardrop R, Brown SJ, Lim EM \& Walsh JP. The relationship between TSH and free T4 in a large population is complex and nonlinear and differs by age and sex. Journal of Clinical Endocrinology and Metabolism 201398 2936-2943. (https://doi.org/10.1210/jc.2012-4223)

23 Yeh NC, Chou CW, Weng SF, Yang CY, Yen FC, Lee SY, Wang JJ \& Tien KJ. Hyperthyroidism and thyroid cancer risk: a population-based cohort study. Experimental and Clinical Endocrinology and Diabetes 2013121 402-406. (https://doi.org/10.1055/s-0033-1341474)

24 Kitahara CM, Rmendiné Farkas DK, Jørgensen JOL, Cronin-Fenton D \& Sørensen HT. Benign thyroid diseases and risk of thyroid cancer: a nationwide cohort study. Journal of Clinical Endocrinology and Metabolism 2018103 2216-2224. (https://doi.org/10.1210/jc.201702599)

25 Mondul AM, Weinstein SJ, Bosworth T, Remaley AT, Virtamo J \& Albanes D. Circulating thyroxine, thyroid-stimulating hormone, and hypothyroid status and the risk of prostate cancer. PLOS ONE 20127 e47730. (https://doi.org/10.1371/journal.pone.0047730)

26 Latteyer S, Christoph S, Theurer S, Hones GS, Schmid KW, Fuhrer D $\&$ Moeller LC. Thyroxine promotes lung cancer growth in an orthotopic mouse model. Endocrine-Related Cancer 201926 565-574. (https://doi.org/10.1530/ERC-18-0353)

27 Lin HY, Tang HY, Shih A, Keating T, Cao G, Davis PJ \& Davis FB. Thyroid hormone is a MAPK-dependent growth factor for thyroid cancer cells and is anti-apoptotic. Steroids $2007 \mathbf{7 2}$ 180-187. (https:// doi.org/10.1016/j.steroids.2006.11.014)

28 Zhang P, Chen L, Song Y, Li X, Sun Y, Xiao Y \& Xing Y. Tetraiodothyroacetic acid and transthyretin silencing inhibit prometastatic effect of L-thyroxin in anoikis-resistant prostate cancer cells through regulation of MAPK/ERK pathway. Experimental Cell Research 2016347 350-359. (https://doi.org/10.1016/j. yexcr.2016.08.019)

29 Theodossiou C, Skrepnik N, Robert EG, Prasad C, Axelrad TW, Schapira DV \& Hunt JD. Propylthiouracil-induced hypothyroidism reduces xenograft tumor growth in athymic nude mice. Cancer 199986 1596-1601. (https://doi.org/10.1002/(SICI)10970142(19991015)86:8<1596::AID-CNCR30>3.0.CO;2-C)

30 Theodossiou C \& Schwarzenberger P. Propylthiouracil reduces xenograft tumor growth in an athymic nude mouse prostate cancer model. American Journal of the Medical Sciences 2000319 96-99. (https://doi.org/10.1097/00000441-200002000-00005) 
$31 \mathrm{Lu}$ C, Zhu X, Willingham MC \& Cheng SY. Activation of tumor cell proliferation by thyroid hormone in a mouse model of follicular thyroid carcinoma. Oncogene 201231 2007-2016. (https://doi. org/10.1038/onc.2011.390)

32 Davis PJ, Goglia F \& Leonard JL. Nongenomic actions of thyroid hormone. Nature Reviews: Endocrinology 201612 111-121. (https:// doi.org/10.1038/nrendo.2015.205)

33 Mu G, Mu X, Xing H, Xu R, Sun G, Dong C, Pan Q \& Xu C. Subclinical hypothyroidism as an independent risk factor for colorectal neoplasm. Clinics and Research in Hepatology and Gastroenterology 201539 261-266. (https://doi.org/10.1016/j.clinre.2014.08.002)

34 L'Heureux A, Wieland DR, Weng CH, Chen YH, Lin CH, Lin TH \& Weng $\mathrm{CH}$. Association between thyroid disorders and colorectal cancer risk in adult patients in Taiwan. JAMA Network Open 20192 e193755. (https://doi.org/10.1001/jamanetworkopen.2019.3755)

35 Boursi B, Haynes K, Mamtani R \& Yang YX. Thyroid dysfunction, thyroid hormone replacement and colorectal cancer risk. Journal of the National Cancer Institute 2015107 djv084. (https://doi. org/10.1093/jnci/djv084)

36 Rennert G, Rennert HS, Pinchev M \& Gruber SB. A case-control study of levothyroxine and the risk of colorectal cancer. Journal of the National Cancer Institute 2010102 568-572. (https://doi.org/10.1093/ jnci/djq042)

37 Friedman GD, Schwalbe JS \& Habel LA. Re: a case-control study of levothyroxine and the risk of colorectal cancer. Journal of the National Cancer Institute 2011103 1637-1639. (https://doi.org/10.1093/jnci/ djr374)

38 Seebacher V, Hofstetter G, Polterauer S, Reinthaller A, Grimm C, Schwameis R, Taucher S, Wagener A, Marth C \& Concin N. Does thyroid-stimulating hormone influence the prognosis of patients with endometrial cancer? A multicentre trial. British Journal of Cancer 2013109 215-218. (https://doi.org/10.1038/bjc.2013.282)

39 Dentice M, Luongo C, Ambrosio R, Sibilio A, Casillo A, Iaccarino A, Troncone G, Fenzi G, Larsen PR \& Salvatore D. Beta-catenin regulates deiodinase levels and thyroid hormone signaling in colon cancer cells. Gastroenterology 2012143 1037-1047. (https://doi. $\operatorname{org} / 10.1053 /$ j.gastro.2012.06.042)

40 Catalano V, Dentice M, Ambrosio R, Luongo C, Carollo R, Benfante A, Todaro M, Stassi G \& Salvatore D. Activated thyroid hormone promotes differentiation and chemotherapeutic sensitization of colorectal cancer stem cells by regulating Wnt and BMP4 signaling. Cancer Research 201676 1237-1244. (https://doi. org/10.1158/0008-5472.CAN-15-1542)

41 Aghajanova L, Stavreus-Evers A, Lindeberg M, Landgren BM, Sparre LS \& Hovatta O. Thyroid-stimulating hormone receptor and thyroid hormone receptors are involved in human endometrial physiology. Fertility and Sterility 201195 230.e1-237.e1. (https://doi. org/10.1016/j.fertnstert.2010.06.079)

42 Ellerhorst JA, Sendi-Naderi A, Johnson MK, Cooke CP, Dang SM \& Diwan AH. Human melanoma cells express functional receptors for thyroid-stimulating hormone. Endocrine-Related Cancer 200613 1269-1277. (https://doi.org/10.1677/erc.1.01239)

43 Murphy E, Glüer CC, Reid DM, Felsenberg D, Roux C, Eastell R \& Williams GR. Thyroid function within the upper normal range is associated with reduced bone mineral density and an increased risk of nonvertebral fractures in healthy euthyroid postmenopausal women. Journal of Clinical Endocrinology and Metabolism 201095 3173-3181. (https://doi.org/10.1210/jc.2009-2630)

44 Leader A, Ayzenfeld RH, Lishner M, Cohen E, Segev D \& Hermoni D. Thyrotropin levels within the lower normal range are associated with an increased risk of hip fractures in euthyroid women, but not men, over the age of 65 years. Journal of Clinical Endocrinology and Metabolism 201499 2665-2673. (https://doi.org/10.1210/jc.20132474)

45 Ndrepepa G, Braun S, Mayer K, Cassese S, Fusaro M, Byrne RA, Hoppmann P, Schukert H, Laugwitz KL \& Kastrati A. Prognostic value of thyroid-stimulating hormone within reference range in patients with coronary artery disease. Metabolism: Clinical and Experimental 201564 1308-1315. (https://doi.org/10.1016/j.metabol.2015.07.009)

46 Li X, Yao Y, Chen Z, Fan S, Hua W, Zhang S \& Fan X. Thyroidstimulating hormone within the normal range and risk of major adverse cardiovascular events in nonischemic dilated cardiomyopathy patients with severe left ventricular dysfunction. Clinical Cardiology 201942 120-128. (https://doi.org/10.1002/ clc.23117)

47 Chen Y, Chen Y, Wang N, Chen C, Nie X, Li Q, Han B \& Lu Y. Thyroid stimulating hormone within the reference range is associated with visceral adiposity index and lipid accumulation product: a population-based study of SPECT-China. Hormone and Metabolic Research 201850 29-36. (https://doi. org/10.1055/s-0043-122235)

48 Brandt J, Borgquist S \& Manjer J. Prospectively measured thyroid hormones and thyroid peroxidase antibodies in relation to risk of different breast cancer subgroups: a Malmo Diet and Cancer Study. Cancer Causes and Control 201526 1093-1104. (https://doi. org/10.1007/s10552-015-0602-8)

49 Franklyn JA, Maisonneuve P, Sheppard M, Betteridge J \& Boyle P. Cancer incidence and mortality after radioiodine treatment for hyperthyroidism: a population-based cohort study. Lancet $19993 \mathbf{3 3}$ 2111-2115. (https://doi.org/10.1016/S0140-6736(98)12295-X)

Received 29 September 2020

Revised version received 8 December 2020

Accepted 12 January 2021 